\title{
TWO APPLICATIONS OF MONOID ACTIONS TO CROSS-SECTIONS
}

\author{
TARA BROUGH, ALAN J. CAIN, AND VICTOR MALTCEV
}

\begin{abstract}
Using a construction that builds a monoid from a monoid action, this paper exhibits an example of a direct product of monoids that admits a prefix-closed regular cross-section, but one of whose factors does not admit a regular cross-section; this answers negatively an open question from the theory of Markov monoids. The same construction is then used to show that for any full trios $\mathfrak{C}$ and $\mathfrak{D}$ such that $\mathfrak{C}$ is not a subclass of $\mathfrak{D}$, there is a monoid with a cross-section in $\mathfrak{C}$ but no cross-section in $\mathfrak{D}$.
\end{abstract}

\section{INTRODUCTION}

Cross-sections (that is, sets of normal forms) of semigroups and groups have been an important area of investigation, both in their own right and in connection with other topics such as automatic structures $\mathrm{ECH}^{+} 92$, CRRT01. Sometimes rather suprising results have emerged, such as the free inverse monoid of rank 1 having no regular cross-section [CS01, Proof of Theorem 2.7].

A group is Markov if it admits a prefix-closed regular language of unique representatives. This notion was introduced by Gromov in his seminal paper on hyperbolic groups [Gro87, § 5.2], and explored further by Ghys \& de la Harpe GdlH90]. The study of the concept of being Markov was extended to semigroups and monoids by the second and third authors [CM14, who proved that the class of Markov monoids is closed under direct product [CM14, Theorem 14.1(1)] and asked whether this class is closed under direct factors CM14, Question 14.6].

One of the main results of this paper is the construction of an example of a monoid that does not have a regular cross-section but whose direct product with the free group of rank 1 does have a regular cross-section, and indeed a prefix-closed one. This proves that neither the class of regular cross-section monoids nor the class of Markov monoids is closed under direct factors. This may shed new light on the long-standing open questions on whether the classes of automatic monoids [CRRT01, Question 6.6] and

The first author was supported by an FCT post-doctoral fellowship (SFRH/BPD/121469/2016). The second author was supported by an Investigador FCT fellowship (IF/01622/2013/CP1161/CT0001). The first and second authors were partially supported by by the Fundação para a Ciência e a Tecnologia (Portuguese Foundation for Science and Technology) through the project UID/MAT/00297/2013 (Centro de Matemática e Aplicações) and the projects PTDC/MHC-FIL/2583/2014 and PTDC/MAT-PUR/31174/2017. The authors thank Ian McQuillan for supplying references on the relationship between ETOL and LIG. The authors thanks the anonymous referee for their careful reading of the paper and many valuable suggestions. 
automatic groups $\mathrm{ECH}^{+} 92$, Open Question 4.1.2] are closed under taking direct factors. (However, the example constructed herein is far from being automatic.)

The other main result is that for any full trios $\mathfrak{C}$ and $\mathfrak{D}$ such that $\mathfrak{C}$ is not a subclass of $\mathfrak{D}$, there is a monoid with a cross-section in $\mathfrak{C}$ but no cross-section in $\mathfrak{D}$.

The main tool is a construction developed by Maltcev \& Ruškuc [MR13] that produces a semigroup from a semigroup action or a monoid from a monoid action; the details of the construction are recalled in Subsection 2.1.

\section{Preliminaries}

2.1. Monoids from monoid actions. This subsection recalls the construction of a monoid from a monoid action introduced (for semigroups) in [MR13, $\S 5]$.

Let $M$ be a monoid acting (from the right) on a set $T$. Define the monoid $M[T]$ to be the disjoint union $M \cup T$ with multiplication in $M$ as before and defined elsewhere by

$$
t m=t \cdot m ; \quad m t=t ; \quad t y=y \quad \text { for all } t, y \in T \text { and } m \in M .
$$

It is straightforward to check that this multiplication is associative.

2.2. Languages and cross-sections. For the definition of basic languagetheoretic concepts, see HU79. Recall in particular that a full trio (also called a cone) is a family of languages, containing at least one non-empty language, closed under homomorphism, inverse homomorphism, and intersection with regular languages. Recall also that full trios are closed under GSM mappings and inverse GSM mappings [HU79, Theorems 11.1 \& 11.2]. Full trios are also closed under the operation of union with a regular language. Since this fact does not seem to be explicitly stated in the literature, we give a proof here:

Lemma 2.1. Full trios are closed under the operation of union with a regular language.

Proof. Let $\mathfrak{C}$ be a full trio, let $L \in \mathfrak{C}$ and let $R$ be a regular language. If $L$ is empty, then $L \cup R$ is regular and so $L \cup R \in \mathfrak{C}$ since a full trio contains all regular languages [HU79, p. 271]. So suppose $L$ is non-empty. Let $A$ be the alphabet of $L \cup R$ and let $x$ be a new symbol not in $A$. Define a homomorphism that fixes each symbol in $A$ and maps $x$ to some word in $L$. Then $L \cup\{x\}$ is also in $\mathfrak{C}$ by the closure of full trios under inverse homomorphism. Now consider the substitution that fixes each symbol in $A$ and maps $x$ to $R$; the image of $L \cup\{x\}$ under this substitution is $L \cup R$. By the closure of full trios under substitution with regular sets HU79, Theorem 11.4], $L \cup R \in \mathfrak{C}$.

For the definitions of tree-adjoining languages, see [Kal10]; for ET0L languages, see [Roz73]; and for indexed languages see [Aho68].

Recall that a cross-section (or set of normal forms) for a monoid $M$ is a language $L$ over some generating set $A$ for $M$ such that every element of $M$ has a unique representative in $L$. Generally, we are interested in crosssections lying in some natural class of languages, which are usually made up 
of languages over a finite alphabet (so that the cross section is over some finite generating set). A Markov monoid is a monoid with a prefix-closed regular cross-section over some (necessarily finite) generating set; see [CM14] for background reading.

Lemma 2.2. Let $M$ be a monoid that has a cross-section in a class of languages $\mathfrak{C}$ that is closed under homomorphisms. Let $A$ be a generating set for $M$. Then $M$ has a cross-section over $A$ in $\mathfrak{C}$.

[This technical result is trivial to prove, but does not seem to appear explicitly in the literature in the form used in this paper, so a proof is supplied.]

Proof. Let $M$ have a cross-section $K$ in $\mathfrak{C}$ with respect to some generating set $B$. For each $b \in B$, choose a word $u_{b} \in A^{*}$ representing $b$. Let $\phi: B^{*} \rightarrow A^{*}$ be the homomorphism that extends the map $b \mapsto u_{b}$. Let $L=\phi(K)$. Then $L$ is clearly also a cross-section for $M$, and, since $\mathfrak{C}$ is closed under homomorphisms, $L$ is also in $\mathfrak{C}$.

\section{DiRECT FACTORS}

This section is dedicated to using the construction described in Subsection 2.1 to define a monoid that does not admit a regular cross-section, but whose direct product with $\mathbb{Z}$ admits a regular cross-section.

Let $F$ be the free monoid with basis $\left\{x, y, y^{\prime}, z, z^{\prime}\right\}$. Let

$$
\begin{aligned}
& P=\left\{p_{\alpha, \beta}: \alpha \in \mathbb{N} \cup\{0\} 0, \beta \in \mathbb{Z}\right\} \\
& T=P \cup\{\Omega\}
\end{aligned}
$$

For convenience, let $\mathbb{B}=\left\{2^{k}: k \in \mathbb{N} \cup\{0\}\right\}$. Define an action of the generators $x, y, y^{\prime}, z, z^{\prime}$ on $T$ as follows:

$$
\begin{gathered}
p_{\alpha, \beta} \cdot x= \begin{cases}p_{\alpha+1,0} & \text { if } \beta=0, \\
\Omega & \text { if } \beta \neq 0 ;\end{cases} \\
p_{\alpha, \beta} \cdot y= \begin{cases}p_{\alpha, \beta+1} & \text { if } \alpha \notin \mathbb{B}, \\
p_{\alpha, 0} & \text { if } \alpha \in \mathbb{B} \text { and } \beta=0, \\
\Omega & \text { if } \alpha \in \mathbb{B} \text { and } \beta \neq 0 ;\end{cases} \\
p_{\alpha, \beta} \cdot y^{\prime}= \begin{cases}p_{\alpha, \beta-1} & \text { if } \alpha \notin \mathbb{B}, \\
p_{\alpha, 0} & \text { if } \alpha \in \mathbb{B} \text { and } \beta=0, \\
\Omega & \text { if } \alpha \in \mathbb{B} \text { and } \beta \neq 0 ;\end{cases} \\
p_{\alpha, \beta} \cdot z= \begin{cases}p_{\alpha, \beta} & \text { if } \alpha \notin \mathbb{B} ; \\
p_{\alpha, \beta+1} & \text { if } \alpha \in \mathbb{B},\end{cases} \\
p_{\alpha, \beta} \cdot z^{\prime}= \begin{cases}p_{\alpha, \beta} & \text { if } \alpha \notin \mathbb{B} . \\
p_{\alpha, \beta-1} & \text { if } \alpha \in \mathbb{B},\end{cases} \\
\Omega \cdot x=\Omega \cdot y=\Omega \cdot y^{\prime}=\Omega \cdot z=\Omega \cdot z^{\prime}=\Omega
\end{gathered}
$$

Figure 1 illustrates the action of $F$ on $T$. 


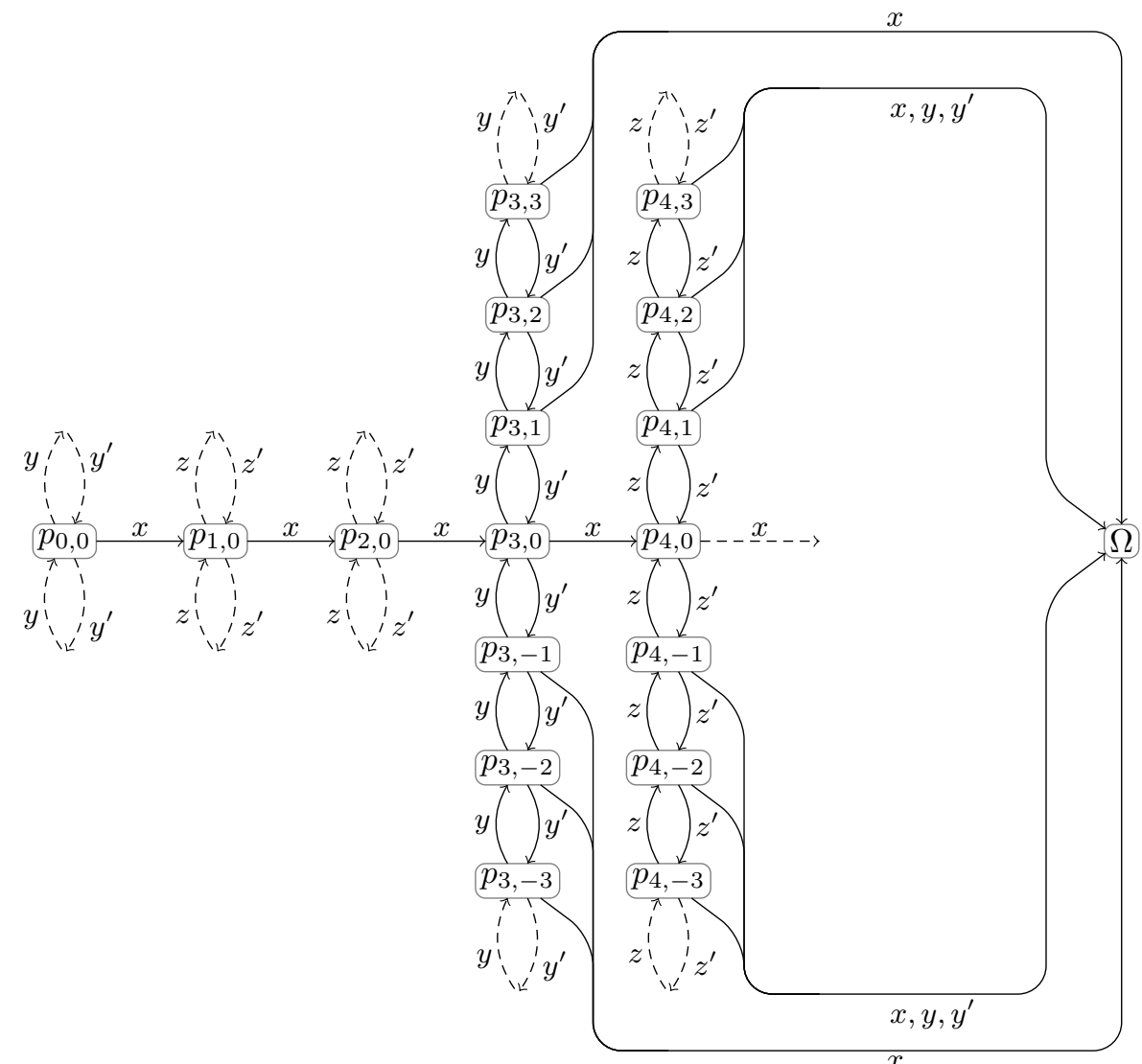

Figure 1. Diagram of the action of $A$ on $T$. Actions which fix points of $T$ (which would be loops at some vertex) are omitted for clarity. The elements $p_{3, \beta}$ and elements $p_{4, \beta}$ are shown to illustrate the different action on $p_{\alpha, \beta}$ when $\alpha$ is and is not a power of 2 .

Proposition 3.1. The direct product $F[T] \times \mathbb{Z}$ has a prefix-closed regular cross-section.

Proof. Let

$$
A=\left\{a, b_{0}, b_{1}, b_{0}^{\prime}, b_{-1}^{\prime}, c_{0}, c_{1}, c_{0}^{\prime}, c_{-1}^{\prime}, d_{1}, d_{-1}, e, f\right\}
$$


where

$$
\begin{array}{rlrl}
a & =(x, 0) & & \\
b_{0} & =(y, 0) & b_{0}^{\prime} & =\left(y^{\prime}, 0\right) \\
b_{1} & =(y, 1) & b_{-1}^{\prime} & =\left(y^{\prime},-1\right) \\
c_{0} & =(z, 0) & c_{0}^{\prime} & =\left(z^{\prime}, 0\right) \\
c_{1} & =(z, 1) & c_{-1}^{\prime} & =\left(z^{\prime},-1\right) \\
d_{1} & =(\varepsilon, 1) & d_{-1} & =(\varepsilon,-1) \\
e & =\left(p_{0,0}, 0\right) & & \\
f & =(\Omega, 0) .
\end{array}
$$

Let

$$
\begin{aligned}
& L_{1}=e a^{*} b_{1}^{*} c_{1}^{*} \cup e a^{*} b_{1}^{*} c_{-1}^{\prime}{ }^{*} \cup e a^{*} b_{-1}^{\prime}{ }^{*} c_{1}^{*} \cup e a^{*} b_{-1}^{\prime}{ }^{*} c_{-1}^{\prime}{ }^{*} \cup f d_{1}^{*} \cup f d_{-1}^{*}, \\
& L_{2}=\left\{a, b_{0}, b_{0}^{\prime}, c_{0}, c_{0}^{\prime}\right\}^{*} d_{1}^{*} \cup\left\{a, b_{0}, b_{0}^{\prime}, c_{0}, c_{0}^{\prime}\right\}^{*} d_{-1}^{*},
\end{aligned}
$$

and let $L=L_{1} \cup L_{2}$. By inspection, the regular language $L$ is prefix-closed.

We claim that $L$ is a regular cross-section for $F[T] \times \mathbb{Z}$. Note first that every word in $L_{1}$ is non-empty and begins with $e$ or $f$, whereas no word in $L_{2}$ begins with $e$ or $f$. Hence the union $L_{1} \cup L_{2}$ is disjoint. The aim is now to show that every element of $T \times \mathbb{Z}$ has a unique representative in $L_{1}$ and that every element of $F \times \mathbb{Z}$ has a unique representative in $L_{2}$.

It is easy to see that $L_{2}$ maps bijectively onto $F \times \mathbb{Z}$, for the prefix in $\left\{a, b_{0}, b_{0}^{\prime}, c_{0}, c_{0}^{\prime}\right\}^{*}$ maps bijectively onto $F \times\{0\}$ while the suffix in $d_{1}^{*} \cup d_{-1}^{*}$ is uniquely determined by the $\mathbb{Z}$-coordinate.

To show that $L_{1}$ maps bijectively onto $T \times \mathbb{Z}$, note first that words of the form $f d_{1}^{*} \cup f d_{-1}^{*}$ map bijectively onto $\{\Omega\} \times \mathbb{Z}$.

It remains to show that the set of words of the form

$$
e a^{\alpha} b_{1}^{\beta} c_{1}^{\gamma}, \quad e a^{\alpha} b_{1}^{\beta} c_{-1}^{\prime}{ }^{\gamma}, \quad e a^{\alpha} b_{-1}^{\prime}{ }^{\beta} c_{1}^{\gamma}, \quad e a^{\alpha} b_{-1}^{\prime}{ }^{\beta} c_{-1}^{\prime}{ }^{\gamma}
$$

maps bijectively onto $P \times \mathbb{Z}$. In each case, the prefix $e a^{\alpha}$ labels a path in the Cayley graph of $F[T]$ that leads to the element $\left(p_{\alpha, 0}, 0\right)$. The paths then stay within the set $\left\{p_{\alpha, \beta}: \beta \in \mathbb{Z}\right\} \times \mathbb{Z}$, but 'look' quite different depending on whether $\alpha \in \mathbb{B}$ (see Figure 2) or $\alpha \notin \mathbb{B}$ (see Figure 3). To prove bijectivity formally, proceed as follows. First, note that

$$
\begin{aligned}
e a^{\alpha} b_{1}^{\beta} c_{1}^{\gamma} & =\left(p_{0,0}, 0\right)(x, 0)^{\alpha}(y, 1)^{\beta}(z, 1)^{\gamma} \\
& =\left(p_{\alpha, 0}, 0\right)(y, 1)^{\beta}(z, 1)^{\gamma} \\
& = \begin{cases}\left(p_{\alpha, \beta}, \beta\right)(z, 1)^{\gamma} & \text { if } \alpha \neq 2^{k} \text { for any } k \\
\left(p_{\alpha, 0}, \beta\right)(z, 1)^{\gamma} & \text { if } \alpha=2^{k} \text { for some } k\end{cases} \\
& = \begin{cases}\left(p_{\alpha, \beta}, \beta+\gamma\right) & \text { if } \alpha \neq 2^{k} \text { for any } k \\
\left(p_{\alpha, \gamma}, \beta+\gamma\right) & \text { if } \alpha=2^{k} \text { for some } k .\end{cases}
\end{aligned}
$$

Similarly,

$$
e a^{\alpha} b_{1}^{\beta} c_{-1}^{\prime}= \begin{cases}\left(p_{\alpha, \beta}, \beta-\gamma\right) & \text { if } \alpha \neq 2^{k} \text { for any } k \\ \left(p_{\alpha,-\gamma}, \beta-\gamma\right) & \text { if } \alpha=2^{k} \text { for some } k\end{cases}
$$




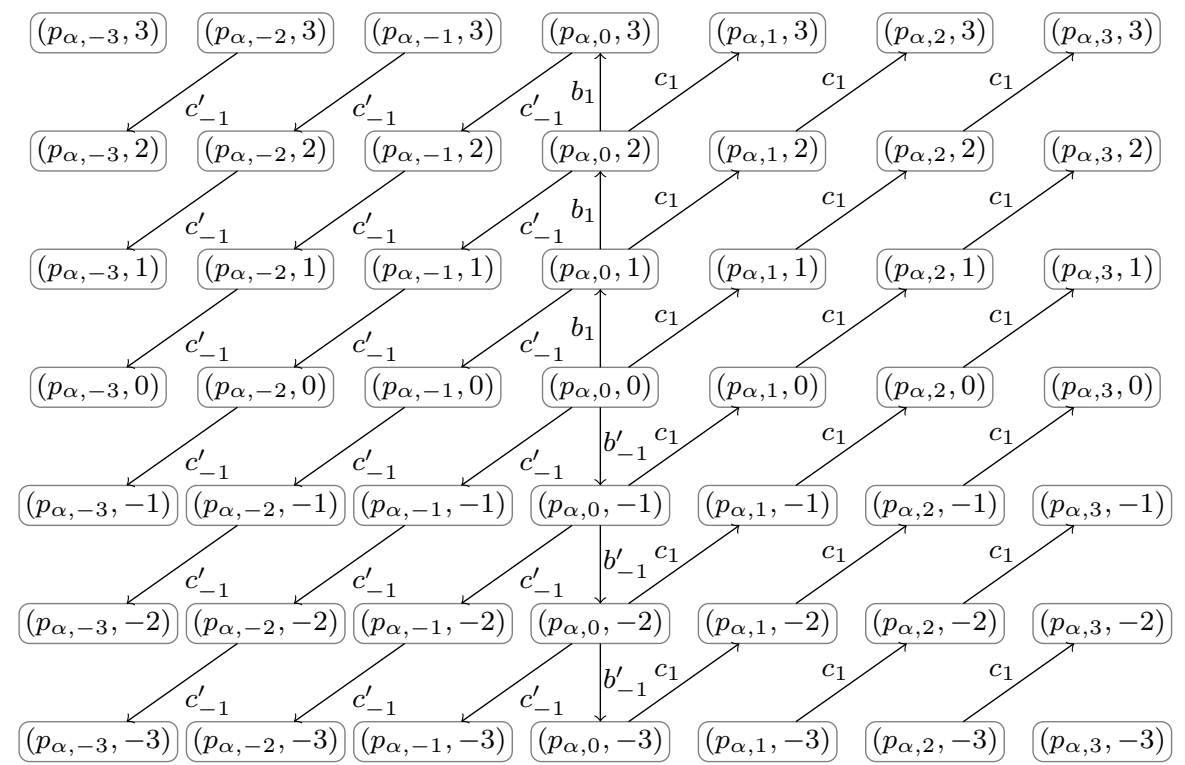

FiguRE 2. Diagram of paths labelled by elements of $L_{1}$ in the Cayley graph of $F[T] \times \mathbb{Z}$, showing the paths passing through the subset $\left\{p_{\alpha, \beta}: \beta \in \mathbb{Z}\right\} \times \mathbb{Z}$, where $\alpha \in \mathbb{B}$.

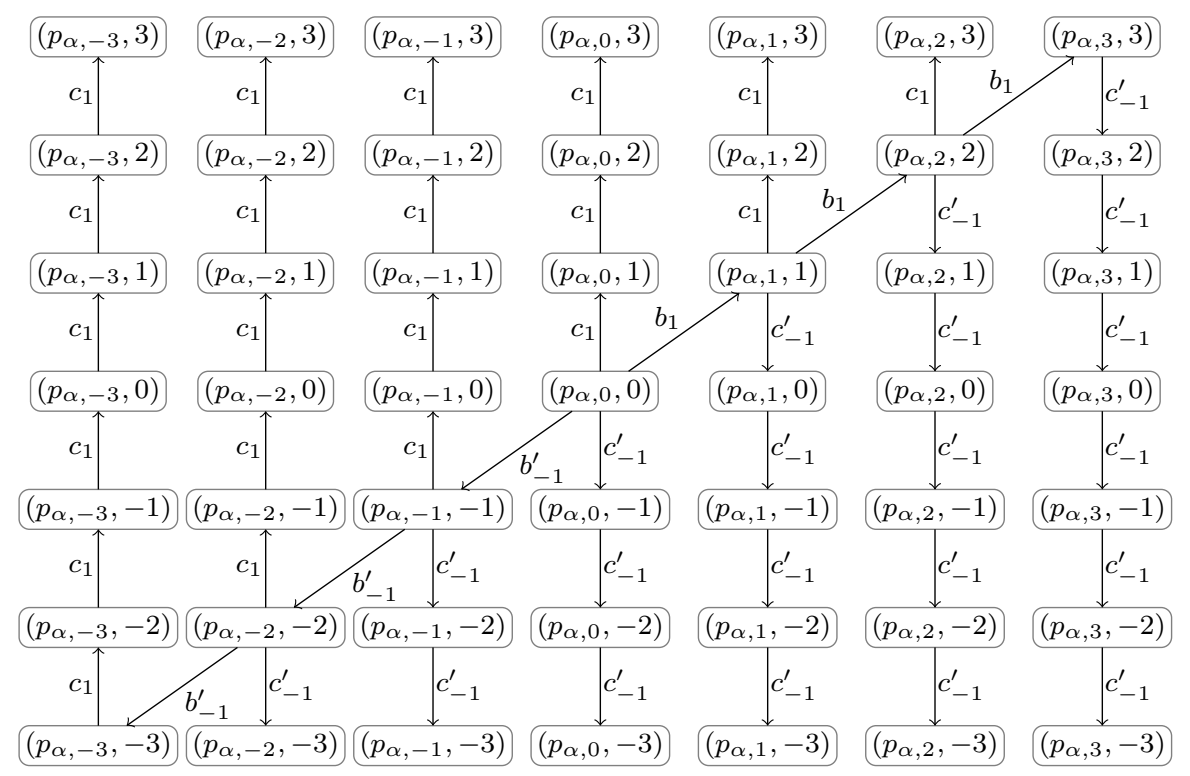

Figure 3. Diagram of paths labelled by elements of $L_{1}$ in the Cayley graph of $F[T] \times \mathbb{Z}$, showing the paths passing through the subset $\left\{p_{\alpha, \beta}: \beta \in \mathbb{Z}\right\} \times \mathbb{Z}$, where $\alpha \notin \mathbb{B}$.

$$
\begin{gathered}
e a^{\alpha} b_{-1}^{\prime}{ }^{\beta} c_{1}^{\gamma}= \begin{cases}\left(p_{\alpha,-\beta},-\beta+\gamma\right) & \text { if } \alpha \neq 2^{k} \text { for any } k \\
\left(p_{\alpha, \gamma},-\beta+\gamma\right) & \text { if } \alpha=2^{k} \text { for some } k\end{cases} \\
e a^{\alpha} b_{-1}^{\prime}{ }^{\beta} c_{-1}^{\prime}{ }^{\gamma}= \begin{cases}\left(p_{\alpha,-\beta},-\beta-\gamma\right) & \text { if } \alpha \neq 2^{k} \text { for any } k \\
\left(p_{\alpha,-\gamma},-\beta-\gamma\right) & \text { if } \alpha=2^{k} \text { for some } k .\end{cases}
\end{gathered}
$$


Let $\left(p_{\alpha, \delta}, \zeta\right) \in T \times \mathbb{Z}$. There are eight cases to consider, depending on whether $\alpha=2^{k}$ for some $k$; whether $\delta \geq 0$ or $\delta<0$; and whether $\zeta \geq \delta$ or $\zeta<0$. We do one exemplary case: $\alpha \neq 2^{k}$ for any $k, \delta \geq 0$, and $\zeta<\delta$. Then

$$
e a^{\alpha} b_{1}^{\delta} c_{-1}^{\prime}{ }^{\delta-\zeta}=\left(p_{\alpha, \delta}, \delta-(\delta-\zeta)\right)=\left(p_{\alpha, \delta}, \zeta\right) .
$$

Furthermore, checking the other cases shows that this is the unique element of $L_{1}$ representing $\left(p_{\alpha, \delta}, \zeta\right)$.

Proposition 3.2. The monoid $F[T]$ does not admit a regular cross-section.

Proof. Suppose for reductio ad absurdum that $F[T]$ admits a regular crosssection. Then by Lemma 2.2, $F[T]$ admits a regular cross-section $L$ over $A=\left\{x, y, y^{\prime}, z, z^{\prime}, p_{0,0}, \Omega\right\}$, since $A$ generates $F[T]$ (because $A$ includes a generating set for $F$ and and the image of $p_{0,0}$ under the action of $F$ is the whole of $T$ ). Since regularity is preserved on replacing a single word, assume that $\Omega \in L$.

For any word $w$ over $A$, one of the following three cases holds:

(1) $p_{0,0}$ and $\Omega$ do not appear in $w$, in which case $w$ represents an element of $F$;

(2) there is an occurrence of $\Omega$ in $w$ such that there is no later occurrence of $p_{0,0}$ in $w$, in which case $w$ represents $\Omega$;

(3) there is an occurrence of $p_{0,0}$ in $w$ such that there is no later occurrence of $\Omega$ in $w$, in which case $w$ represents the same element of $F[T]$ as the suffix of $w$ starting with the last occurrence of $p_{0,0}$.

Let $L^{(1)}$ be the language consisting of suffixes of words in $L$ that lie in $p_{0,0}\left\{x, y, y^{\prime}, z, z^{\prime}\right\}^{*}$. Note that $L^{(1)}$ can be obtained by applying a GSM mapping to $L$, and so $L^{(1)}$ is regular. (The required GSM initially reads input symbols without producing output. It non-deterministically chooses some point at which to produce output, and subsequently outputs each symbol it reads, checking that the suffix after the chosen point is of the required form.) Note also that $\Omega \in L \backslash L^{(1)}$. All words in $L$ that have suffixes in $L^{(1)}$ satisfy case 3 above. Thus the language $L^{(1)}$ must map bijectively to $T \backslash\{\Omega\}$.

Let $p_{0,0} v \in L^{(1)}$. Suppose that $v=v^{\prime} x w x v^{\prime \prime}$, where $w \in\left\{y, y^{\prime}, z, z^{\prime}\right\}^{*}$. Then $v^{\prime} x$ must send $p_{0,0}$ to some $p_{\alpha, 0}$ (for the symbol $x$ would send any other point to $\Omega$, contradicting the fact that $\Omega \in L$ is a unique representative). Further, if $p_{\alpha, 0} \cdot w=p_{\alpha, \beta}$ for $\beta \neq 0$ (note that the action of $w$ cannot alter $\alpha$ ), then $p_{0,0} \cdot v=p_{\alpha, \beta} \cdot x v^{\prime \prime}=\Omega$, which is again a contradiction. Hence $p_{\alpha, 0} \cdot w=p_{\alpha, 0}$. Thus deleting the subword $w$ from $v$ does not alter the represented element. Similar reasoning applies if $v=w x v^{\prime \prime}$ for some $w \in\left\{y, y^{\prime}, z, z^{\prime}\right\}^{*}$; again $w$ can be deleted without altering the represented element. Apply a GSM mapping to $L^{(1)}$ that deletes any string of symbols from $\left\{y, y^{\prime}, z, z^{\prime}\right\}$ between $p_{0,0}$ and $x$ or between two symbols $x$; this yields a regular language $L^{(2)} \subseteq p_{0,0} x^{*}\left\{y, y^{\prime}, z, z^{\prime}\right\}$ mapping bijectively to $T \backslash\{\Omega\}$. (The required GSM initially outputs each symbol it reads. On encountering a symbol $p_{0,0}$ or $x$, it non-deterministically guesses whether there now follows a string of symbols from $\left\{y, y^{\prime}, z, z^{\prime}\right\}$ followed by another $x$. If it guesses 'yes', it checks whether what follows is a string of this form, producing no output until it reaches the $x$, when it resumes outputting each symbol it reads. If 
it guesses 'no', it continues to output each symbol it reads, checking that what follows is not a string of the given form.)

Now let $p_{0,0} v \in L^{(2)}$. Suppose that $v=v^{\prime} y w y v^{\prime \prime}$ for $w \in\left\{z, z^{\prime}\right\}$ and that $p_{0,0} \cdot v^{\prime} y=p_{\alpha, \beta}$. If $\alpha \notin \mathbb{B}$, then $p_{\alpha, \beta} \cdot w=p_{\alpha, \beta}$ and so deleting $w$ does not alter the represented element. So assume $\alpha \in \mathbb{B}$. Then $p_{0,0} \cdot v^{\prime}=$ $p_{0,0} \cdot v^{\prime} y=p_{\alpha, 0}$, since otherwise the action of $y$ would lead to $\Omega$. Similarly, $p_{0,0} \cdot v^{\prime} y w=p_{0,0} \cdot v^{\prime} y w y=p_{\alpha, 0}$. So $w$ fixes $p_{0,0} \cdot v^{\prime} y$ and so deleting $w$ does not alter the represented element.

The same reasoning applies if one replaces either or both of the distinguished symbols $y$ by $y^{\prime}$. Apply a GSM mapping to $L^{(2)}$ that deletes any string of symbols from $\left\{z, z^{\prime}\right\}$ between two symbols from $\left\{y, y^{\prime}\right\}$; this yields a regular language $L^{(3)} \subseteq p_{0,0} x^{*}\left\{z, z^{\prime}\right\}^{*}\left\{y, y^{\prime}\right\}^{*}\left\{z, z^{\prime}\right\}^{*}$ that maps bijectively to $T \backslash\{\Omega\}$. (The required GSM functions similarly to the last-described one.)

Let $p_{0,0} v \in L^{(3)}$. Suppose that $v=x^{\alpha} w y v^{\prime \prime}$ for $w \in\left\{z, z^{\prime}\right\}^{*}$. Suppose $p_{0,0} \cdot x^{\alpha} w=p_{\alpha, \beta}$. Then $\beta=0$, for otherwise the action of $y$ would lead to $\Omega$. Hence $w$ fixes $p_{0,0} \cdot x^{\alpha}$ and so deleting $w$ does not alter the represented element. The same reasoning applies if one replaces the distinguished symbol $y$ by $y^{\prime}$. Apply a GSM mapping to $L^{(3)}$ that deletes any string of symbols from $\left\{z, z^{\prime}\right\}$ between $p_{0,0}$ and a symbol from $\left\{y, y^{\prime}\right\}$; this yields a regular language $L^{(4)} \subseteq p_{0,0} x^{*}\left\{y, y^{\prime}\right\}^{*}\left\{z, z^{\prime}\right\}^{*}$ that maps bijectively to $T \backslash\{\Omega\}$. (Again, the required GSM functions similarly to the last-described one.)

Let $n$ be larger than the number of states in an automaton recognizing $L^{(4)}$. Consider $p_{2^{n}, n} \in T$. Let $p_{0,0} x^{2^{n}} v w \in L^{(4)}$, where $v \in\left\{y, y^{\prime}\right\}^{*}$ and $w \in\left\{z, z^{\prime}\right\}^{*}$, be the unique word representing $p_{2^{n}, n}$. Then since at least $n$ symbols $z$ are required to reach $p_{2^{n}, n}$ from $p_{2^{n}, 0}$, the word $w$ has length at least $n$

By the pumping lemma, $p_{0,0} x^{2^{n}-k} v w \in L^{(4)}$ for some $k$ such that $0<$ $k<n$. Let $p_{\alpha, \beta}=p_{0,0} x^{2^{n}-k} v$; then $\alpha=2^{n}-k \notin \mathbb{B}$, so that $z$ and $z^{\prime}$ fix $p_{\alpha, \beta}$. Since $w$ has length at least $n$, it factors as $w=p q r$ such that $p_{0,0} x^{2^{n}-k} v p q^{i} r \in L^{(4)}$ for all $i \in \mathbb{N} \cup\{0\}$. But $z$ and $z^{\prime}$ fix $p_{\alpha, \beta}$, so all these words represent $p_{\alpha, \beta}$, which contradicts the fact that $L^{(4)}$ maps bijectively onto $T \backslash\{\Omega\}$.

Combining Propositions 3.1 and 3.2 yields the following result:

Theorem 3.3. (1) The class of monoids with regular cross-sections is not closed under taking direct factors.

(2) The class of Markov monoids is not closed under taking direct factors.

However, $F[T]$ has a cross-section in the next natural language class that strictly contains the regular languages:

Proposition 3.4. $F[T]$ has a one-counter cross-section.

Proof. Let $A=\left\{x, y, y^{\prime}, z, z^{\prime}, p_{0,0}, \Omega\right\}$ and let $L$ be the one-counter language

$$
\left\{x, y, y^{\prime}, z, z^{\prime}\right\}^{*} \cup p_{0,0} x^{*}\left\{y^{n} z^{n},\left(y^{\prime}\right)^{n}\left(z^{\prime}\right)^{n}: n \in \mathbb{N}\right\} .
$$


Clearly, $\left\{x, y, y^{\prime}, z, z^{\prime}\right\}^{*}$ maps bijectively onto $F$. Further, $p_{0,0} \cdot x^{\alpha} y^{n} z^{n}=$ $p_{\alpha, n}$ and $p_{0,0} \cdot x^{\alpha}\left(y^{\prime}\right)^{n}\left(z^{\prime}\right)^{n}=p_{\alpha,-n}$ (regardless of whether $\alpha \in \mathbb{B}$ ), and so $p_{0,0} x^{*}\left\{y^{n} z^{n},\left(y^{\prime}\right)^{n}\left(z^{\prime}\right)^{n}: n \in \mathbb{N}\right\}$ maps bijectively onto $T$.

\section{Cross-SeCtions in Different Classes of LANGUages}

This section is dedicated to showing that we can 'separate' two full trios, one not contained in the other, using monoid cross-sections, and applying this result to some particular interesting language classes.

Theorem 4.1. Let $\mathfrak{C}$ and $\mathfrak{D}$ be full trios such that $\mathfrak{C}$ is not a subclass of $\mathfrak{D}$. Then there is a monoid with a cross-section in $\mathfrak{C}$ but no cross-section in $\mathfrak{D}$.

Proof. Since $\mathfrak{C}$ is not a subclass of $\mathfrak{D}$, there is a language $K$ over some finite alphabet $B$ that is in $\mathfrak{C}$ but not in $\mathfrak{D}$. Since full trios always contain the empty language, $K$ is thus non-empty. Let

$$
T=\left\{p_{u}: u \in B^{*}\right\} \cup\left\{q_{u}: u \in K\right\} \cup\{\Omega\} .
$$

Let $z$ be a new symbol not in $B$, let $F$ be the free monoid on $B \cup\{z\}$, and let $F$ act on $T$ as follows

$$
\begin{aligned}
& p_{u} \cdot b=p_{u b} \quad \text { for } u \in B^{*} \text { and } b \in B \text {; } \\
& p_{u} \cdot z=\left\{\begin{array}{ll}
q_{u} & \text { if } u \in K, \\
\Omega & \text { if } u \notin K,
\end{array} \quad \text { for } u \in B^{*} ;\right. \\
& q_{u} \cdot x=\Omega \quad \text { for } u \in K \text { and } x \in B \cup\{z\} \text {; } \\
& \Omega \cdot x=\Omega \quad \text { for } x \in B \cup\{z\} .
\end{aligned}
$$

The aim is now to show that the monoid $F[T]$ has a cross-section in $\mathfrak{C}$ but not in $\mathfrak{D}$.

Let $A=B \cup\left\{z, p_{\varepsilon}, \Omega\right\}$; note that $A$ is a generating set for $F[T]$. Let

$$
L=(B \cup\{z\})^{*} \cup p_{\varepsilon} B^{*} \cup p_{\varepsilon} K z .
$$

Deletion of a fixed prefix $p_{\varepsilon}$ and a fixed suffix $z$ can be performed by a GSM mapping. Since full trios are closed under inverse GSM mappings, and since $K$ is in $\mathfrak{C}$, it follows that $p_{\varepsilon} K z$ is also in $\mathfrak{C}$. Hence, by the closure of full trios under the operation of union with a regular language (Lemma 2.1), $L$ is in $\mathfrak{C}$. Finally, it is clear that $L$ is a cross-section for $F[T]$ since the sublanguage $(B \cup\{z\})^{*}$ maps bijectively onto $F$, the sublanguage $p_{\varepsilon} B^{*}$ maps bijectively onto $\left\{p_{u}: u \in B^{*}\right\}$, and the sublanguage $p_{\varepsilon} K z$ maps bijectively onto $\left\{q_{u}: u \in K\right\}$, and these three sublanguages, whose union is $L$, are disjoint.

Now suppose for reductio ad absurdum that $F[T]$ admits a cross-section in $\mathfrak{D}$. By Lemma 2.2, assume without loss of generality that the cross-section is a language $L$ over $B \cup\left\{z, p_{\varepsilon}, \Omega\right\}$. Since replacing a single word in $L$ can be carried out via intersection with a regular language and then union with a (one-element) regular language (see Lemma 2.1), assume that $\Omega \in L$.

Let $L^{\prime}$ be the language consisting of suffixes of words in $L$ that lie in $p_{\varepsilon} v$, where $v \in(B \cup\{z\})^{*}$. Note that $L^{\prime}$ can be obtained by applying a GSM mapping to $L$, and so $L^{\prime} \in \mathfrak{D}$. (The GSM that extracts the required suffixes functions similarly to the one in the proof of Proposition 3.2.) By definition of multiplication in $F[T]$, and noting that $\Omega \in L \backslash L^{\prime}$, the words 


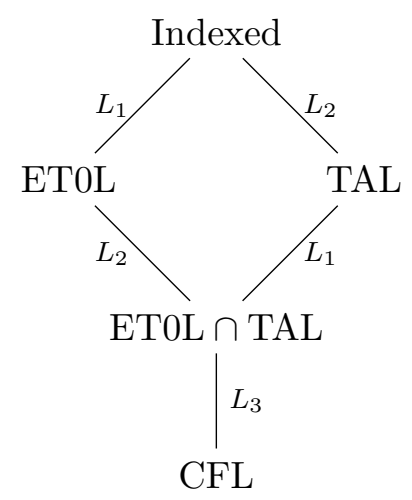

Figure 4. Containment of selected classes of languages.

in $L^{\prime}$ must map bijectively to $T \backslash\{\Omega\}$. By definition of the action of $F$ on $T$, the words in $L^{\prime}$ that map bijectively to $\left\{q_{u}: u \in K\right\}$ are precisely those of the form $p_{\varepsilon} u z$, where $u \in K$. Intersection with a regular language gives the language of the words $u$ such that $p_{\varepsilon} u z \in L^{\prime}$, showing that $K \in \mathfrak{D}$. But this contradicts the fact that $K \notin \mathfrak{D}$. Hence $F[T]$ does not have a cross-section in $\mathfrak{D}$.

Each of the following language classes forms a full trio: the indexed languages Aho68, Theorem 3.2]; ET0L languages Roz73, Theorem 18]; the tree-adjoining languages (TAL) [Kal10, p. 59]; context-free languages (CFL) [HU79, p. 278].

Every ET0L language is indexed [Cul74, Corollary 4.1], and the class of TALs is equivalent to the languages recognised by a restricted type of indexed grammars called linear indexed grammars [Kal10, p. 72], hence the TALs are also indexed. The class of context-free languages is contained in both the ET0L languages [Roz73] and in the TALs [Kal10, p. 58]. (These containments are illustrated in Figure 4, )

The aim is now to show that, for each containment $\mathfrak{D} \subsetneq \mathfrak{C}$ in Figure 4 , there is a monoid with a cross-section in $\mathfrak{C}$ but that does not admit a crosssection in $\mathfrak{D}$. By Theorem 4.1, it suffices to exhibit a language that is in $\mathfrak{C}$ but not in $\mathfrak{D}$.

Corollary 4.2. There exists a monoid that admits a TAL (and hence indexed) cross-section but does not admit an ETOL cross-section.

Proof. Let $L \subseteq X^{*}$ be any context-free but non-EDT0L language (the EDT0L languages are a proper subclass of the ETOL languages (note the 'D' in the name of the subclass); for the existence of context-free non-EDT0L languages, see [ER77]). Let $X^{\prime}$ be a copy of $X$ and $\phi: X^{*} \rightarrow\left(X^{\prime}\right)^{*}$ the homomorphism defined by $x \phi=x^{\prime}$. Then the language $L_{1}=\left\{w(w \phi)^{\mathrm{rev}}: w \in L\right\}$ is generated by a linear indexed grammar [DP84, Corollary 3.6] (note that the notion of linear indexed grammar in DP84 is more restricted than the one in [Kal10] equivalent to tree-adjoining grammars) and is hence a TAL and therefore also indexed. However, $L_{1}$ is not ET0L [ERS76]. The result follows by Theorem 4.1. 
Corollary 4.3. There exists a monoid that admits an ETOL (and hence indexed) cross-section but admits no TAL cross-section.

Proof. Let $L_{2}=\left\{w w w: w \in\{a, b\}^{*}\right\}$. Then $L_{2}$ is generated by an ET0Lsystem with 4 tables, each containing a single non-trivial production. The non-trivial productions are $S \rightarrow T T T, T \rightarrow a T, T \rightarrow b T$, and $T \rightarrow \varepsilon$. However, $L_{2}$ is not a TAL [Kal10, Lemma 4.15]. The result follows by Theorem 4.1.

Corollary 4.4. There exists a monoid that admits a cross-section that is both ETOL and TAL but admits no context-free cross-section.

Proof. Finally, let $L_{3}=\left\{a^{n} b^{n} c^{n}: n \in \mathbb{N}\right\}$. Then $L_{3}$ is TAL Kal10, Problem 4.1] and ETOL, since it is generated by the following 3-table ETOLsystem:

$$
\begin{aligned}
& S \rightarrow A B C ; \\
& A \rightarrow a, \quad B \rightarrow b, \quad C \rightarrow c ; \\
& A \rightarrow \varepsilon, \quad B \rightarrow \varepsilon, \quad C \rightarrow \varepsilon .
\end{aligned}
$$

However, $L_{3}$ is easily shown not to be context-free by a pumping argument. The result follows by Theorem 4.1.

\section{REFERENCES}

[Aho68] A. V. Aho. 'Indexed Grammars - An Extension of Context-Free Grammars'. Journal of the ACM, 15, no. 4 (1968), pp. 647-671. DOI: $10.1145 / 321479.321488$

[CM14] A. J. Cain \& V. Maltcev. 'Markov semigroups, monoids, and groups'. International Journal of Algebra and Computation, 24, no. 5 (2014), pp. 609-653. DOI: $10.1142 / \mathrm{S} 021819671450026 \mathrm{X}$

[CRRT01] C. M. Campbell, E. F. Robertson, N. Ruškuc, \& R. M. Thomas. 'Automatic semigroups'. Theoretical Computer Science, 250, no. 1-2 (2001), pp. 365-391. DOI: 10.1016/S0304-3975(99)00151-6

[CS01] A. Cutting \& A. Solomon. 'Remarks concerning finitely generated semigroups having regular sets of unique normal forms'. Journal of the Australian Mathematical Society, 70, no. 03 (2001), p. 293. DOI: 10.1017/S1446788700002354.

[Čul74] K. Čulik. 'On some families of languages related to developmental systems'. International Journal of Computer Mathematics, 4, no. 1-4 (1974), pp. 31-42. DOI: $10.1080 / 00207167408803079$.

[DP84] J. Duske \& R. Parchmann. 'Linear indexed languages'. Theoretical Computer Science, 32, no. 1-2 (1984), pp. 47-60. Dor: 10.1016/0304-3975(84)90023-9.

$\left[\mathrm{ECH}^{+} 92\right]$ D. B. A. Epstein, J. W. Cannon, D. F. Holt, S. V. F. Levy, M. S. Paterson, \& W. P. Thurston. Word Processing in Groups. Jones \& Bartlett, Boston, MA, 1992.

[ER77] A. Ehrenfeucht \& G. Rozenberg. 'On some context free languages that are not deterministic ETOL languages'. RAIRO Informatique théorique, 11, no. 4 (1977), pp. 273-291. DOI: 10.1051/ita/1977110402731.

[ERS76] A. Ehrenfeucht, G. Rozenberg, \& S. Skyum. 'A Relationship between ET0L and EDT0L languages'. Theoretical Computer Science, 1, no. 4 (1976), pp. 325-330. DOI: 10.1016/0304-3975(76)90076-1.

[GdlH90] E. Ghys \& P. de la Harpe. 'La propriété de Markov pour les groupes hyperboliques'. In E. Ghys \& P. de la Harpe, eds, Sur les Groupes Hyperboliques d'après Mikhael Gromov, no. 83 in Progress in Mathematics, chapter 9, pp. 165-187. Birkhäuser, Boston, 1990. 
[Gro87] M. Gromov. 'Hyperbolic Groups'. In S. M. Gersten, ed., Essays in Group Theory, no. 8 in Mathematical Sciences Research Institute Publications, pp. 75-263. Springer-Verlag, 1987.

[HU79] J. E. Hopcroft \& J. D. Ullman. Introduction to Automata Theory, Languages, and Computation. Addison-Wesley, Reading, MA, 1st edition, 1979.

[Kal10] L. Kallmeyer. Parsing Beyond Context-Free Grammars. Cognitive Technologies. Springer, 2010.

[MR13] V. Maltcev \& N. Ruskuc. 'On hopfian cofinite subsemigroups'. 2013. arXiv: 1307.6929 .

[Roz73] G. Rozenberg. 'Extension of Tabled 0L-Systems and Languages'. International Journal of Computer 63 Information Sciences, 2, no. 4 (1973), pp. 311-336. DOI: $10.1007 / \mathrm{BF} 00985664$

Centro de Matemática e Aplicações, Faculdade de Ciências e Tecnologia, Universidade Nova de Lisboa, 2829-516 Caparica, Portugal

E-mail address: t.brough@fct.unl.pt

Centro de Matemática e Aplicações, Faculdade de Ciências e Tecnologia, Universidade Nova de Lisboa, 2829-516 Caparica, Portugal

E-mail address: a.cain@fct.unl.pt

C/O SECOND AUTHOR

E-mail address: victor.maltcev@googlemail.com 